\title{
Green Finishing of Cotton Fabrics Using Xylitol-Extended Citric Acid Cross-linking System on a Pilot Scale
}

Jian Liu ${ }^{a, b}$, Bijia Wang ${ }^{a, b}$, Xiaomei Xu ${ }^{a, b}$, Jiangang Chen ${ }^{a, b}$, Luyi Chen ${ }^{a, b}$ and Yiqi $\operatorname{Yang}^{c, d *}$

${ }^{\mathrm{a}}$ Key Laboratory of Science and Technology of Eco-Textiles, Ministry of Education, Donghua University, 2999 North Renmin Road, Shanghai 201620, China

${ }^{\mathrm{b}}$ College of Chemistry, Chemical Engineering and Biotechnology, Donghua University, 2999 North Renmin Road, Shanghai 201620, China

${ }^{\mathrm{c}}$ Department of Textiles, Merchandising and Fashion Design, HECO Building, University of Nebraska-Lincoln, Lincoln, NE 68583-0802, United States

${ }^{\mathrm{d}}$ Department of Biological Systems Engineering and Nebraska Center for Materials and Nanoscience, HECO Building, University of Nebraska-Lincoln, Lincoln NE 68583-0802, United States 
Table S1. Central Composite Design Matrix of Five Experimental Variables and the Three Measurable Responses

\begin{tabular}{|c|c|c|c|c|c|c|c|c|}
\hline \multirow{2}{*}{$\begin{array}{l}\text { Run } \\
\text { Order }\end{array}$} & \multirow{2}{*}{$\begin{array}{c}\mathrm{T} \\
(\min )\end{array}$} & \multirow{2}{*}{$\begin{array}{l}\text { Temp. } \\
\left({ }^{\circ} \mathrm{C}\right)\end{array}$} & \multirow{2}{*}{$\begin{array}{c}\mathrm{CA} \\
(\mathrm{g} / \mathrm{L})\end{array}$} & \multirow{2}{*}{$\begin{array}{l}\text { Xyl. } \\
(\mathrm{g} / \mathrm{L})\end{array}$} & \multirow{2}{*}{$\begin{array}{c}\mathrm{P} \\
\left(\mathrm{kg} / \mathrm{m}^{2}\right)\end{array}$} & \multicolumn{3}{|c|}{ Responses } \\
\hline & & & & & & WI & WRA $\left(^{\circ}\right)$ & $\mathrm{TS}(\mathrm{cN})$ \\
\hline 1 & 2 & 175 & 100 & 27.5 & 2 & 61.7 & 238.5 & 770 \\
\hline 2 & 3 & 160 & 125 & 20 & 3 & 66.8 & 243.3 & 693.7 \\
\hline 3 & 3 & 160 & 125 & 20 & 3 & 67.7 & 241.5 & 730.7 \\
\hline 4 & 4 & 145 & 100 & 12.5 & 2 & 75.1 & 198.1 & 1188.2 \\
\hline 5 & 2 & 175 & 100 & 27.5 & 4 & 66.7 & 224.5 & 919.5 \\
\hline 6 & 4 & 145 & 100 & 12.5 & 4 & 75.2 & 195.3 & 1263.1 \\
\hline 7 & 4 & 175 & 150 & 12.5 & 2 & 48.7 & 262.3 & 555.4 \\
\hline 8 & 4 & 145 & 150 & 12.5 & 4 & 74.1 & 222 & 1000.2 \\
\hline 9 & 4 & 175 & 100 & 12.5 & 4 & 62 & 241.8 & 769.7 \\
\hline 10 & 2 & 145 & 150 & 27.5 & 2 & 76 & 198.5 & 1147.2 \\
\hline 11 & 2 & 145 & 100 & 27.5 & 2 & 76 & 209.1 & 1570.5 \\
\hline 12 & 4 & 145 & 150 & 27.5 & 4 & 72.9 & 212.6 & 957.5 \\
\hline 13 & 5 & 160 & 125 & 20 & 3 & 63.8 & 247.8 & 735.9 \\
\hline 14 & 3 & 160 & 125 & 20 & 3 & 68.8 & 240.1 & 752.3 \\
\hline 15 & 4 & 175 & 100 & 27.5 & 4 & 61.8 & 241.3 & 717.8 \\
\hline 16 & 3 & 160 & 125 & 20 & 3 & 67.4 & 243.8 & 781.8 \\
\hline 17 & 2 & 145 & 150 & 27.5 & 4 & 75.7 & 199.1 & 1226.8 \\
\hline 18 & 2 & 175 & 100 & 12.5 & 2 & 62.5 & 245.1 & 761.4 \\
\hline 19 & 3 & 160 & 125 & 35 & 3 & 69.6 & 223.8 & 848.3 \\
\hline 20 & 4 & 175 & 100 & 12.5 & 2 & 59.6 & 256.3 & 745.5 \\
\hline 21 & 3 & 130 & 125 & 20 & 3 & 77.1 & 201.5 & 1591.1 \\
\hline 22 & 3 & 160 & 125 & 20 & 3 & 67.9 & 248.8 & 782.6 \\
\hline 23 & 3 & 160 & 175 & 20 & 3 & 63.7 & 242 & 704.9 \\
\hline 24 & 4 & 145 & 150 & 12.5 & 2 & 71 & 238.5 & 767.5 \\
\hline 25 & 2 & 175 & 100 & 12.5 & 4 & 63.4 & 238.6 & 849.5 \\
\hline 26 & 2 & 175 & 150 & 12.5 & 4 & 51.9 & 263.3 & 592.9 \\
\hline 27 & 2 & 175 & 150 & 27.5 & 2 & 56 & 254 & 615.1 \\
\hline 28 & 4 & 145 & 150 & 27.5 & 2 & 73.7 & 212.3 & 892 \\
\hline 29 & 4 & 175 & 150 & 12.5 & 4 & 49.8 & 267.6 & 552.7 \\
\hline 30 & 3 & 160 & 125 & 20 & 5 & 67 & 234.8 & 835.9 \\
\hline 31 & 3 & 160 & 125 & 20 & 3 & 66 & 244.1 & 779.7 \\
\hline 32 & 3 & 160 & 75 & 20 & 3 & 71.8 & 206.3 & 1142.9 \\
\hline 33 & 2 & 175 & 150 & 27.5 & 4 & 59.1 & 254.1 & 649.1 \\
\hline 34 & 2 & 145 & 150 & 12.5 & 4 & 74.9 & 224.3 & 1100.7 \\
\hline
\end{tabular}




\begin{tabular}{ccccccccc}
35 & 3 & 160 & 125 & 5 & 3 & 65.4 & 222.6 & 872.3 \\
36 & 2 & 145 & 100 & 12.5 & 2 & 76.2 & 193.8 & 1618.3 \\
37 & 4 & 145 & 100 & 27.5 & 4 & 75.4 & 209.3 & 1419.7 \\
38 & 2 & 145 & 150 & 12.5 & 2 & 74.1 & 220 & 1107.6 \\
39 & 2 & 145 & 100 & 12.5 & 4 & 76.8 & 187.6 & 1583.5 \\
40 & 3 & 190 & 125 & 20 & 3 & 47.8 & 268.5 & 558.8 \\
41 & 4 & 175 & 100 & 27.5 & 2 & 64.7 & 247.8 & 674.5 \\
42 & 4 & 175 & 150 & 27.5 & 4 & 53.2 & 265.1 & 547.6 \\
43 & 3 & 160 & 125 & 20 & 3 & 68 & 244.3 & 783.7 \\
44 & 3 & 160 & 125 & 20 & 3 & 67.2 & 242.6 & 802.6 \\
45 & 4 & 175 & 150 & 27.5 & 2 & 51.8 & 264.3 & 512.6 \\
46 & 2 & 145 & 100 & 27.5 & 4 & 76.3 & 191.8 & 1627.4 \\
47 & 2 & 175 & 150 & 12.5 & 2 & 52.5 & 261.8 & 608 \\
48 & 1 & 160 & 125 & 20 & 3 & 74.8 & 217.8 & 1134.4 \\
49 & 3 & 160 & 125 & 20 & 1 & 64 & 248.5 & 627 \\
50 & 4 & 145 & 100 & 27.5 & 2 & 75.7 & 203.3 & 1331.7 \\
\hline
\end{tabular}

T, time; Temp., temperature; Xyl., xylitol; P, padder-roll pressure 
Table S2. Costs and Effectiveness of Low Formaldehyde 2D Resin (F-ECO) and Selected Formaldehyde-free DP Resins Crosslinking Systems

\begin{tabular}{|c|c|c|c|c|}
\hline Finishing agent & $\begin{array}{c}\text { Cost } \\
(\$ / \mathrm{kg} \text { fabrics })\end{array}$ & WI & WRA & $\begin{array}{c}\text { Effectiveness } \\
\text { Score }^{\mathrm{a}}\end{array}$ \\
\hline $\mathrm{CA}$ & 0.18 & 68 & 269 & 4 \\
\hline BTCA & 0.90 & 72 & 270 & 4.5 \\
\hline $\mathrm{NF}$ & 0.45 & 68 & 214 & 2.3 \\
\hline WFF & 1.63 & 71 & 261 & 4 \\
\hline SRD787 & 0.37 & 70 & 238 & 3 \\
\hline F-ECO & 0.34 & 74 & 283 & 5 \\
\hline
\end{tabular}

${ }^{a}$ Calculated as the average of the ranking scores for WI and WRA performances, which are determined according to Table S2. 
Table S3. Ranking Score Criteria for WRA and WI

\begin{tabular}{lccccccc}
\hline Ranking score & 5 & 4.5 & 4 & 3.5 & 3 & 2 & 1 \\
\hline WI & $\geq 74$ & $\geq 72$ & $\geq 70$ & $\geq 68$ & $\geq 60$ & $\geq 55$ & $<50$ \\
WRA & $\geq 275$ & $\geq 265$ & $\geq 255$ & $\geq 245$ & $\geq 240$ & $\geq 220$ & $<220$ \\
\hline
\end{tabular}

